\title{
Estratégia para redução e fixação de fraturas mandibulares complexas. Um relato de
}

\section{caso}

Strategy for reduction and fixation of complex mandibular fractures. A case report

Estrategia de reducción y fijación de fracturas mandibulares complejas. Reporte de un caso

Recebido: 28/07/2021 | Revisado: 04/08/2021 | Aceito: 18/08/2021 | Publicado: 21/08/2021

Leonardo Alan Delanora

ORCID: https://orcid.org/0000-0002-3002-4420 Universidade Estadual Paulista Júlio Mesquita Filho, Brasil

E-mail: leonardoaland@gmail.com

Julio Cesar Silva de Oliveira

ORCID: https://orcid.org/0000-0003-2796-5059

Universidade Estadual Paulista Júlio Mesquita Filho, Brasil

E-mail: Oliveirajulius@yahoo.com.br

Tiburtino José de Lima Neto

ORCID: https://orcid.org/0000-0002-8297-4057 Universidade Estadual Paulista Júlio Mesquita Filho, Brasil

E-mail:tiburtinoneto@hotmail.com

Mirela Caroline Silva

ORCID: https://orcid.org/0000-0002-9455-3807 Universidade Estadual Paulista Júlio Mesquita Filho, Brasil

E-mail: mirela.c.silva@unesp.br

Nathália Januario de Araujo

ORCID: https://orcid.org/0000-0003-1812-7723

Universidade Estadual Paulista Júlio Mesquita Filho, Brasil

E-mail: nathaliajanuario@outlook.br

Idelmo Rangel Garcia-Junior

ORCID: https://orcid.org/0000-0001-8892-781X Universidade Estadual Paulista Júlio Mesquita Filho, Brasil E-mail: idelmo.rangel@unesp.br

Leonardo Perez Faverani

ORCID: https://orcid.org/0000-0003-2249-3048 Universidade Estadual Paulista Júlio Mesquita Filho, Brasil E-mail: Leonardo.faverani@unesp.br

\section{Resumo}

Fraturas mandibulares são relativamente comuns, porém os padrões de fratura e as modalidades de tratamento variam conforme as características anatômicas e biomecânicas da região. O presente artigo tem por objetivo realizar um relato de caso de um paciente de 80 anos, vítima de queda da própria altura, cursando com fratura mandibular atípica em região de corpo e ângulo mandibular esquerdo, com três traços de fratura se unindo na região posterior de ramo mandibular. O tratamento foi instituído conforme o padrão de fratura observado e seguindo os princípios de fixação interna rígida, com boa evolução pós-operatória, mesmo que o paciente ainda se apresente em acompanhamento. Com isso, pode-se concluir que se faz necessário o manejo adequado de qualquer padrão de fratura e uma proservação adequada de cada caso para diminuição da incidência de complicações pós-operatórias.

Palavras-chave: Fixação interna de fraturas; Mandíbula; Procedimentos cirúrgicos operatórios.

\begin{abstract}
Mandibular fractures are relatively common, but fracture patterns and treatment modalities vary depending on the anatomical and biomechanical characteristics of the region. This article aims to carry out a case report of an 80-yearold patient, victim of a fall from his own height, with an atypical mandibular fracture in the left mandibular body and angle region, with three fracture lines uniting in the posterior region of mandibular branch. Treatment was instituted according to the observed fracture pattern and following the principles of rigid internal fixation, with good postoperative evolution, even if the patient is still being followed up. Thus, it can be concluded that the proper management of any fracture pattern and an adequate follow-up of each case is necessary to reduce the incidence of postoperative complications.
\end{abstract}

Keywords: Fracture fixation interna; Mandible; Surgical procedures, operative. 


\begin{abstract}
Resumen
Las fracturas mandibulares son relativamente comunes, pero los patrones de fractura y las modalidades de tratamiento varían según las características anatómicas y biomecánicas de la región. Este artículo tiene como objetivo realizar un reporte de caso de un paciente de 80 años, víctima de una caída desde su propia altura, con una fractura mandibular atípica en la región del cuerpo y ángulo mandibular izquierda, con tres líneas de fractura unidas en la región posterior. de la rama mandibular. El tratamiento se instituyó de acuerdo con el patrón de fractura observado y siguiendo los principios de la fijación interna rígida, con buena evolución postoperatoria, incluso si el paciente aún está en seguimiento. Así, se puede concluir que el manejo adecuado de cualquier patrón de fractura y un seguimiento adecuado de cada caso es necesario para reducir la incidencia de complicaciones postoperatorias.
\end{abstract}

Palabras clave: Fijación interna de fracturas; Mandíbula; Procedimientos quirúrgicos operativos.

\title{
1. Introdução
}

A mandíbula é o único osso móvel da face e participa de diversas funções básicas como fonação, deglutição, mastigação, além da manutenção da oclusão. Apesar da anatomia óssea, a mandíbula está propensa a fraturas por ser um arco aberto e estar localizada mais anteriormente na face, sendo uma região proeminente. As fraturas de mandíbula apresentam uma etiologia dependente de alguns fatores, como condições demográficas, culturais, socioeconômicas, além de apresentarem uma alta incidência em acidentes automobilísticos, agressões físicas e quedas. Constituem ainda a $4^{\mathrm{a}}$ causa mais comum de fratura facial, sendo a região de côndilo a mais acometida (Andrade, Limoeiro, Souza, Nascimento, \& Moreira, 2021; Rampaso, Mattioli, Andrade Sobrinho, \& Rapoport, 2009; Sever, Kulahci, Uygur, \& Karagoz, 2011).

Dentre os principais sinais e sintomas que podem estar associados a uma fratura mandibular tem-se a má oclusão, lacerações, hematomas e equimoses; perda de dentes e/ou crepitação, alteração no movimento mandibular, trismo e alargamento do terço inferior da face. O tipo de fratura e o deslocamento do fragmento variam conforme o trauma e a ação da musculatura na região, sendo que traumas de alta intensidade podem levar a fraturas mais cominutivas e regiões com alta atuação da musculatura podem apresentar-se com fraturas mais deslocadas, sendo ambas situações mais desfavoráveis ao tratamento (Ladeinde, Adeyemo, Ogunlewe, \& Gbotolorun, 2009; Malik, Debnath, Adhyapok, \& Hazarika, 2017; Matteini \& Belli, 2001; Meneses-Santos et al., 2020).

As fraturas na região de ângulo mandibular podem ocorrer associadas a diversas outras fraturas, sendo na região contralateral de parassínfise as mais comuns e com menor incidência na forma cominutiva. Fraturas atípicas na região de ângulo e corpo requerem maior atenção durante tratamento devido a posição anatômica e biomecânica da mandíbula do ângulo dificultarem o tratamento de fraturas nesta região, tendo uma incidência de complicações pós-operatórias de 0 a 32\% (AlMoraissi \& Ellis III, 2014; Ibrahim et al., 2020; Ladeinde et al., 2009; Mackenzie, Grosse, Barnard, \& Brennan, 2006; Sever et al., 2011).

Entretanto, o tratamento com redução aberta e fixação interna rígida dos fragmentos ósseos da fratura são os métodos utilizados para restabelecimento da função e estética e tem se mostrado eficazes mesmo em casos de fraturas atípicas. Por isso, se faz importante o correto manejo dessas fraturas, mesmo se utilizando técnicas convencionais de tratamento, para diminuir a incidência de complicações (MacKay et al., 2017; Mackenzie et al., 2006; Paza, Abuabara, \& Passeri, 2008; Sever et al., 2011; Ushin, Levenets, Paniotov, \& Pristrom, 1988).

O objetivo deste artigo é realizar um relato de caso clínico de uma fratura mandibular atípica abrangendo a região de corpo e ângulo mandibular esquerdo, com três traços de fratura se unindo na região posterior de ramo mandibular.

\section{Metodologia}

Trata-se de um estudo retrospectivo, descritivo e observacional no formato de Relato de Caso Clínico de um paciente tratado em ambiente hospitalar. Os dados epidemiológicos, história da doença e registros fotográficos foram coletados através do prontuário físico e eletrônico, após autorização dos responsáveis pelo paciente, mediante a assinatura do Termo de 
Consentimento Livre e Esclarecido (TCLE), seguindo os preceitos para publicação sem a identificação do paciente e conforme os princípios éticos da Declaração de Helsinque. Como meio de complemento e embasamento do estudo, foram feitas buscas na literatura utilizando o MeSH Database. Com base na busca, foi feita uma breve discussão conforme o que foi encontrado na literatura, descrito o diagnóstico e tratamento, visando os melhores resultados para o paciente.

\section{Relato de Caso}

Paciente masculino, 80 anos, vítima de queda da própria altura, cursando com trauma em região de face, ao exame físico extraoral, foi constatado edema e equimose em região de corpo e ângulo mandibular esquerdo que seguia até região inferior do pescoço à esquerda e mais anterior abaixo do mento. A palpação, leve degrau ósseo palpável na região de corpo/ângulo mandibular esquerdo. Limitação de abertura bucal a princípio por queixa álgica. Ao exame intraoral não foram observadas alterações dignas de nota.

Ao exame de imagem foi constatado três traços de fratura que se uniam na parte posterior do ramo mandibular esquerdo, dois delas se originavam da parte basal da mandíbula e um deles da porção anterior do ramo, do mesmo lado, como pode ser observado na Figura 1.

Figura 1: (A) Vista extraoral do paciente com edema e equimose em região de mandíbula esquerda, equimose se estendendo até região anteroinferior do mento e inferior a osso hióide. (B) Reconstrução 3D evidenciando traços de fratura em região de corpo mandibular, ângulo e ramo mandibular esquerdo.
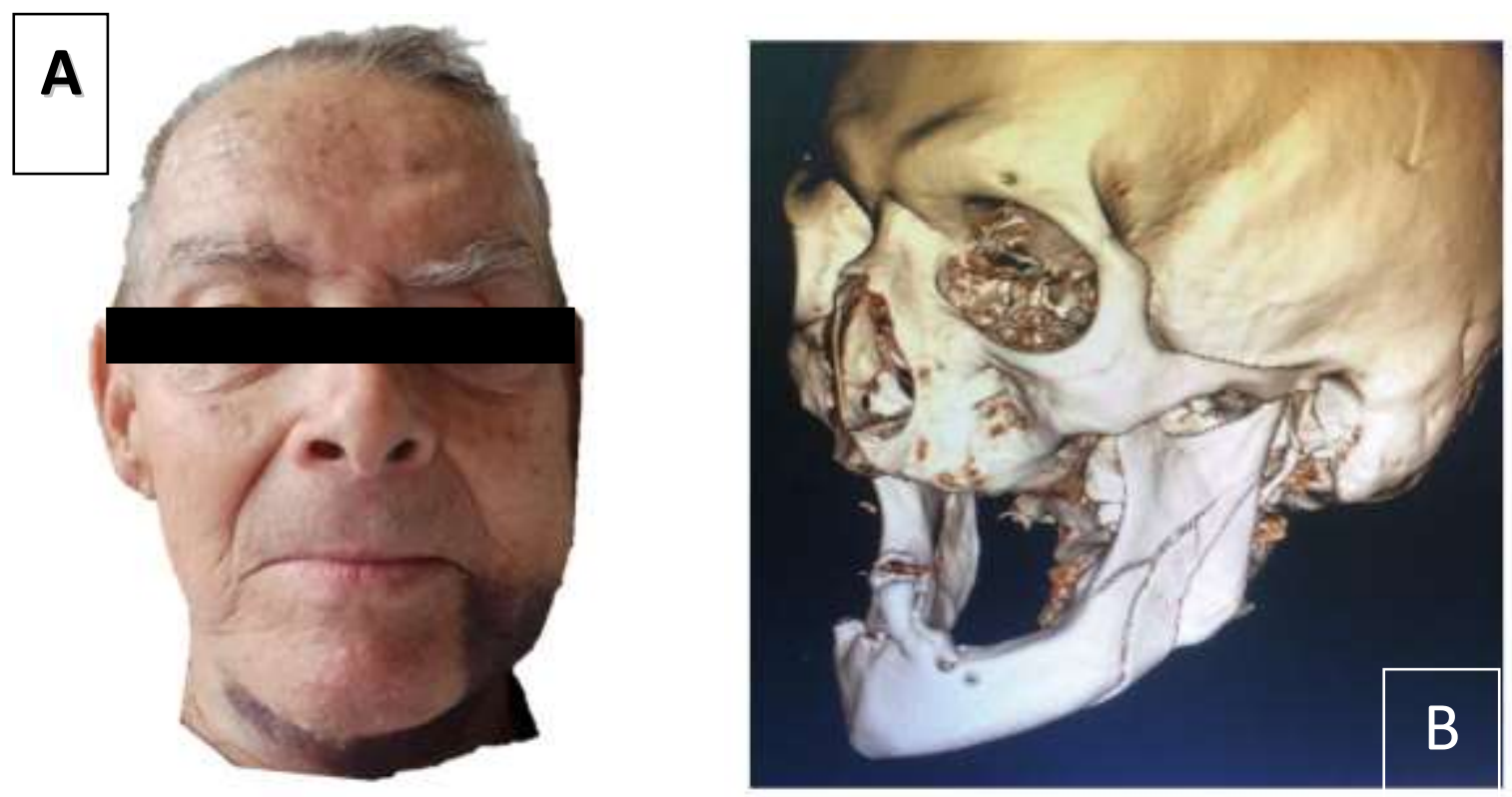

Fonte: Autores (2021).

Na Figura 1, é possível visualizar o edema e equimose na região do impacto que resultou na fratura, na porção B, é possível evidenciar os três traços de fratura distintos, onde se encontram na região posterior de ramo mandibular, tendo três pontos de partida distintos: porção anterior do ramo mandibular e dois traços da porção de corpo/ângulo mandibular.

Devido ao degrau ósseo e instabilidade da fratura foi optado pela redução e fixação das fraturas com placas e parafusos de titânio, Figura 2. 
Foram realizados um acesso intraoral, para a redução e fixação da fratura em região anterior de ramo mandibular e duas outras pequenas incisões extra orais para inserção do trocater e fixação da porção mais basal da fratura.

Foi utilizado uma placa em "X" para fixação anterior com 4 parafusos e duas placas retas, uma com 5 furos e outra com 10 furos. Além disso, foram utilizados dois parafusos lag screw para estabilização da fratura em ramo anterior, complementando a placa em "X", como pode ser visto na Figura 2.

Figura 2: (A) Acesso intraoral evidenciando os traços de fratura, em especial do na região anterior do ramo mandibular esquerdo. (B) Fixação com placas de titânio e parafusos já em posição e fratura estabilizada. É possível notar o trocater em posição para acesso as placas de fixação laterais.

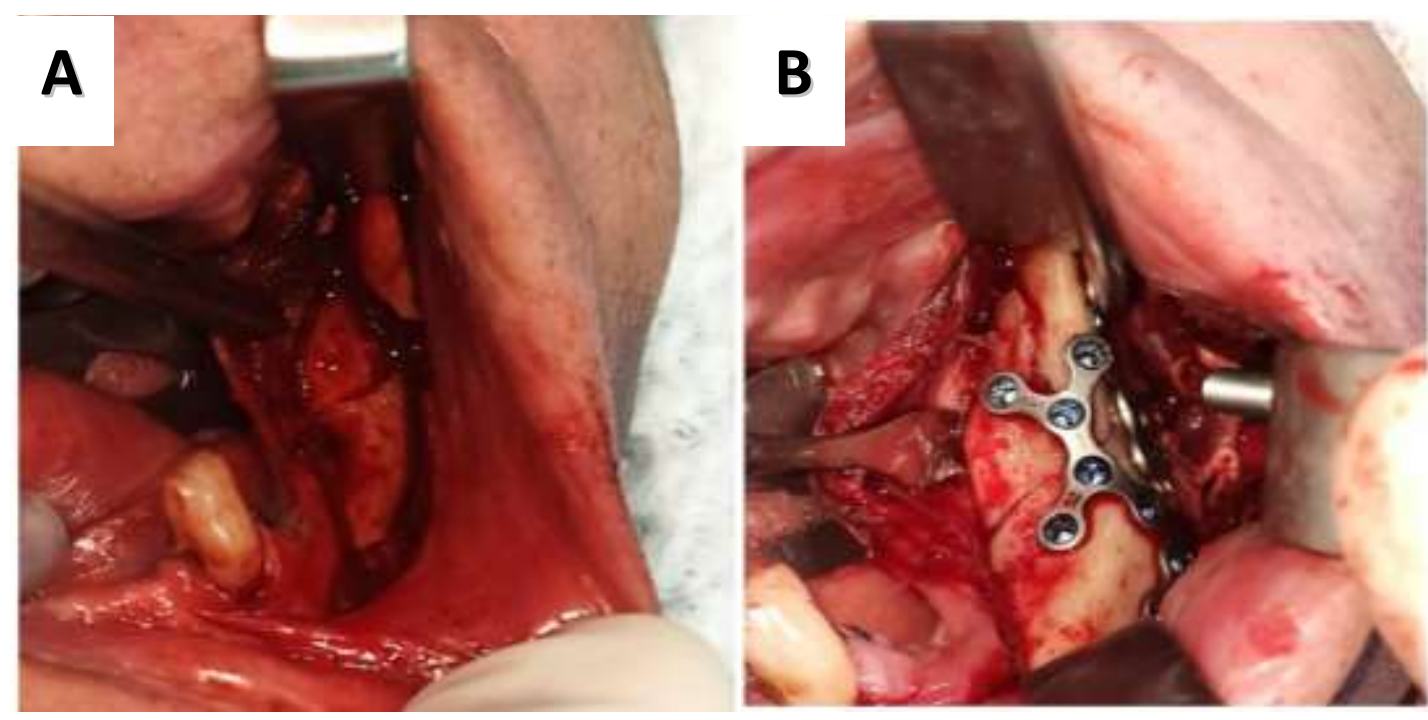

Fonte: Autores (2021).

Na Figura 2 é possível observar os traços de fratura após acesso intraoral, evidenciando a cominuição da mesma (A) e após a osteossíntese com a placa geométrica em X na zona de tensão, evidenciando uma boa coaptação dos fragmentos ósseos (B).

Sendo finalizado com pontos reabsorvíveis (Vicryl 4-0), com pontos de segurança de nylon 5-0 em região intraoral e nylon 4-0 na região extraoral (acesso do trocater). Visível na Figura 3. 
Figura 3: (A) Acesso intraoral suturado com fio de nylon 5-0 e vicryl 5-0. (B) Dois acessos extraorais que foram utilizados para uso do trocater suturados com fio nylon 4-0.

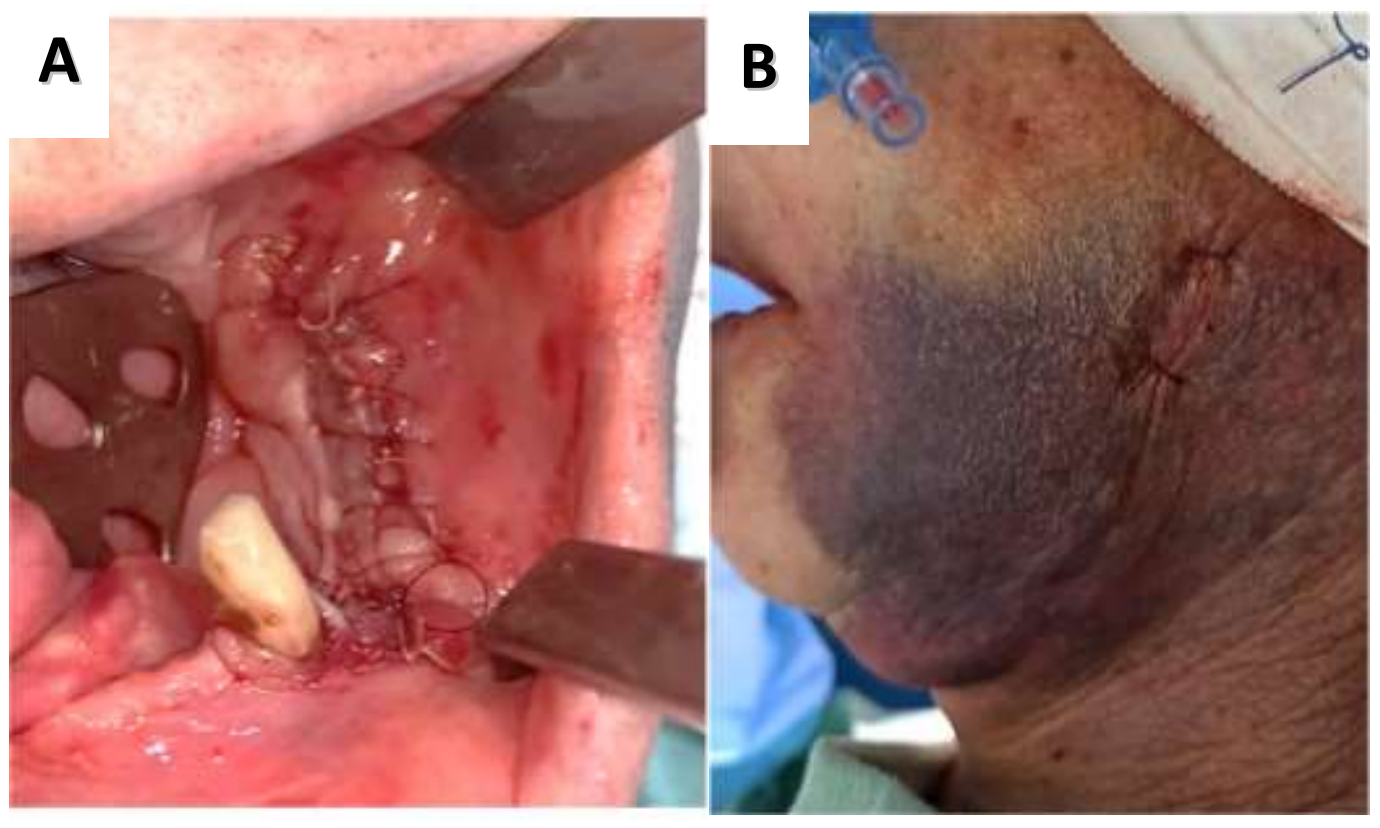

Fonte: Autores (2021).

Na Figura 3 é possível observar o aspecto pós-operatório imediato, logo após a síntese de tecidos moles, tanto da região intraoral (A) quanto da região extraoral (B). Equimose e edema observado na região extraoral é referente ao trauma sofrido pelo paciente.

O paciente no pós-operatório apresentou edema compatível com o procedimento, sem queixas álgicas e com bom aspecto cicatricial. Ele permanece em acompanhamento e sem sinais de infecção, inflamação ou fadiga das placas. Na TC é possível observar a estabilização e fixação satisfatória da fratura, como visto na Figura 4. 
Figura 4: (A) Vista extra oral notando edema e equimose pós-operatório compatível com o procedimento. (B) Reconstrução 3D mostrando a estabilização e fixação da fratura com as placas e parafusos em posição.
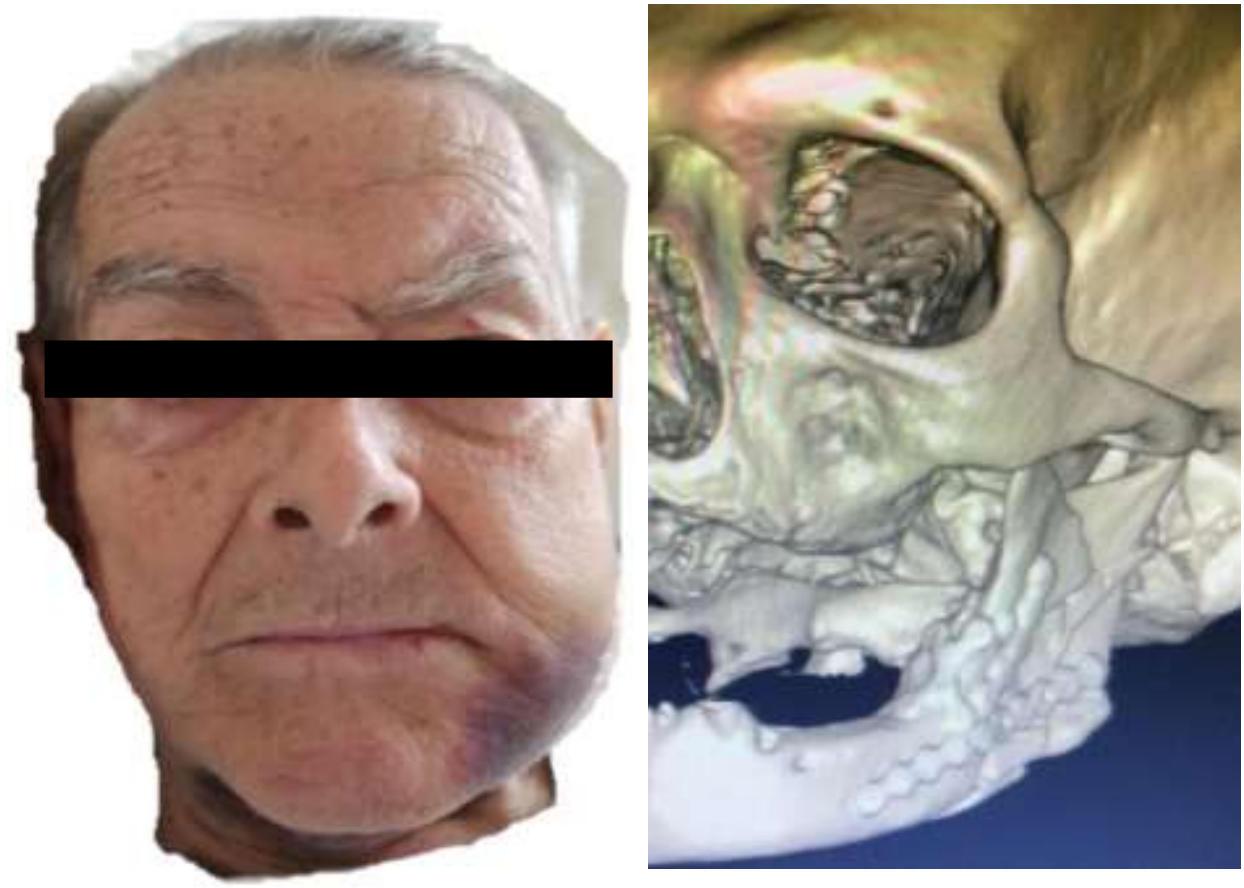

Fonte: Autores (2021).

Na Figura 4 é possível observar o aspecto extraoral pós-operatório do paciente (A), edema e equimose compatíveis com o procedimento cirúrgico e a reconstrução 3D que evidencia as placas em posição e um bom reposicionamento dos fragmentos ósseos mesmo com a cominuição da fratura (B).

\section{Discussão}

Segundo Rampaso et al. (2009) pacientes edêntulos totais ou parciais, idosos, são mais propensos a fraturas na região maxilofacial devido à ausência de dentes para amortecer o impacto, por conta disso, é necessário ter uma maior atenção no manejo e tratamento desses pacientes uma vez que a fratura está presente. A região de ângulo e corpo mandibular apresentam ação biomecânica do músculo masseter e pterigoideo medial podendo apresentar fraturas favoráveis ou desfavoráveis conforme a direção da linha de fratura, além disso, as fraturas de ângulo representam 30\% de todas as fraturas mandibulares (Al-Moraissi \& Ellis III, 2014; Junior et al., 2019; Rampaso et al., 2009). No caso descrito, o paciente se tratava de um idoso de 80 anos de idade, porém com pequeno deslocamento entre os segmentos, devido a ação biomecânica favorável na região.

Há ainda na literatura grandes discussões acerca de tratamento de fraturas abrangendo região de ângulo e ramo mandibular devido a aspectos como a anatomia da região, com mudanças abruptas de curvatura e pela presença de terceiros molares. Porém, está bem consolidado que o tratamento de fraturas nessas regiões deve ter por objetivo anular as forças de tensão, na borda superior da mandíbula, e compressão, na borda inferior. Sendo a redução aberta e a fixação interna rígida as modalidades eficientes e mais utilizadas com essa finalidade (Oliveira et al., 2021; Paza et al., 2008; Ramalho, Araújo, Santos, Caubi, \& Sobreira, 2011; Torres et al., 2020). Segundo Junior et al. (2019) variações dentro dessas categorias de tratamento podem existir dependendo do tipo de fratura, da abordagem escolhida para osteossíntese e o método de fixação.

No caso descrito, optou-se por realizar algumas mudanças na fixação interna rígida devido à complexidade dos traços das fraturas, porém seguindo os mesmos princípios. Utilizou-se uma placa em X na zona de tensão, uma placa reta de 10 furos 
na zona de compressão e uma placa reta de 5 furos em uma região intermediária devido a presença de linha de fratura seguindo posteriormente para borda posterior de ramo mandibular. Além disso, 2 parafusos lag screw para estabilização de fratura na região anterior de ramo para complementar a placa em X. Mesmo que seja pouco descrito na literatura, Ramalho et al. (2011) evidencia o uso de parafusos lag screw para região de ângulo e corpo mandibular, inclusive para fraturas cominutivas como forma final de tratamento e, até mesmo, simplificação da fratura para aplicação de placas de reconstrução (Ibrahim et al., 2020; Ramalho et al., 2011; Souza et al., 2018; Wubulihasimu et al., 2019). Além disso, Al-Moraissi and Ellis III (2014) mostrou que o uso de placas geométricas na fixação de fraturas de ângulo diminuiu em 58\% o risco de complicações pós-operatórias em comparação ao uso de mini placas padrão.

Morris et al. (2015) mostrou não existir um padrão de fratura mandibular e evidenciou que a análise dessas lesões não se limita apenas aos componentes anatômicos e funcionais que até aqui foram explorados, entretanto envolvem inúmeras outras variáveis que ainda não foram completamente exploradas na literatura. Por ainda não existir uma modalidade de tratamento para cada padrão de fratura encontrado, é importante realizar a proservação dos pacientes após a instituição do tratamento justamente para prevenir qualquer tipo de complicação pós-operatória, já que há uma grande incidência de complicações relacionadas a esse tipo de fratura (Junior et al., 2019; Ladeinde et al., 2009; Morris, Bebeau, Brockhoff, Tandon, \& Tiwana, 2015). O paciente evolui bem no período pós-operatório, mantendo a estabilidade promovida pela síntese, com ausência de sinais de infecção ou exposição das placas utilizadas para osteossíntese da fratura.

\section{Conclusão}

Dessa forma, pode-se concluir que o manejo e o tipo de tratamento são individualizados conforme cada caso devido à ausência de padrões nas fraturas mandibulares, desde que os princípios da fixação interna rígida sejam mantidos e os pacientes tenham um acompanhamento a longo prazo para prevenção de possíveis complicações. Mesmo que a fratura descrita seja atípica obteve-se um resultado satisfatório, sem qualquer comprometimento estético e/ou funcional para o paciente.

\section{Referências}

Al-Moraissi, E. A., \& Ellis III, E. (2014). What method for management of unilateral mandibular angle fractures has the lowest rate of postoperative complications? A systematic review and meta-analysis. Journal of oral and maxillofacial surgery, 72(11), $2197-2211$.

Andrade, M. J. d. H., Limoeiro, A. G. d. S., Souza, C. C., Nascimento, W. M., \& Moreira, D. C. (2021). Epidemiological study of facial fractures in a brazilian subpopulation. Research, Society and Development, 10(5), e27910514937. doi:10.33448/rsd-v10i5.14937

Ibrahim, G., Gaetti-Jardim, E., Mendonça, J., Oliveira, J., Pelissaro, G., Silva, J., \& Santos, A. (2020). Tratamento cirúrgico de fratura bilateral de mandíbula. Perspectivas Experimentais e Clínicas, Inovações Biomédicas e Educação em Saúde (PECIBES), 6(1), 18-18.

Junior, W. d. S. F., Fanderuff, M., Reis, T. V. R., Rebellato, N. L. B., Scariot, R., \& Klüppel, L. E. (2019). Atypical mandibular angle fracture. Journal of Craniofacial Surgery, 30(2), e155-e157.

Ladeinde, A., Adeyemo, W., Ogunlewe, M., \& Gbotolorun, O. (2009). An unusual fracture of the mandible. Ghana medical journal, $43(4), 179$.

MacKay, J. W., Murray, P. J., Kasmai, B., Johnson, G., Donell, S. T., \& Toms, A. P. (2017). Subchondral bone in osteoarthritis: association between MRI texture analysis and histomorphometry. Osteoarthritis and cartilage, 25(5), 700-707.

Mackenzie, N., Grosse, S., Barnard, J., \& Brennan, P. (2006). INTERESTING CASE: An unusual fracture of the angle of the mandible. British Journal of Oral and Maxillofacial Surgery, 44(1), 48.

Malik, K., Debnath, S. C., Adhyapok, A. K., \& Hazarika, K. (2017). An Atypical Variant of Superolateral Dislocation of the Mandibular Condyle: A Case Report. Journal of oral and maxillofacial surgery, 75(10), 2183. e2181-2183. e2186.

Matteini, C., \& Belli, E. (2001). An unusual case of sub-condylar bilateral fracture and bilateral post-traumatic temporomandibular ankylosis. Minerva stomatologica, 50(9-10), 337-342.

Meneses-Santos, D., Oliveira, M. M. M., Sol, I., Rodrigues, C. M. d. C., Lima, F. G. G. P., Silva, C. J., \& Silva, M. C. P. d. (2020). Surgical treatment of comminuted mandible fracture involving simplification with miniplates: Report of two cases. Research, Society and Development, 9(12), e43991211398. doi:10.33448/rsd-v9i12.11398 
Research, Society and Development, v. 10, n. 10, e599101018815, 2021

(CC BY 4.0) | ISSN 2525-3409 | DOI: http://dx.doi.org/10.33448/rsd-v10i10.18815

Morris, C., Bebeau, N. P., Brockhoff, H., Tandon, R., \& Tiwana, P. (2015). Mandibular fractures: an analysis of the epidemiology and patterns of injury in 4,143 fractures. Journal of oral and maxillofacial surgery, 73(5), 951. e951-951. e912.

Oliveira, M. M. M., Almeida, A. C. d., Lauand, G. A., Santos, D. M., Silva, C. J., \& Lima, F. G. G. P. (2021). Surgical treatment of bilateral fracture of atrophic mandibula: Case report. Research, Society and Development, 10(1), e11110111531. doi:10.33448/rsd-v10i1.11531

Paza, A. O., Abuabara, A., \& Passeri, L. A. (2008). Analysis of 115 mandibular angle fractures. Journal of oral and maxillofacial surgery, 66(1), 73-76.

Ramalho, R. d. A., Araújo, F. A. d. C., Santos, F. S. M. d., Caubi, A. d. F., \& Sobreira, T. (2011). Tratamento de fratura de mandíbula: miniplacas e parafu sos $\mathrm{x}$ lag screws-relato de caso. Revista de Cirurgia e Traumatologia Buco-maxilo-facial, 11(1), 59-63.

Rampaso, C. L., Mattioli, T. M. F., Andrade Sobrinho, J. d., \& Rapoport, A. (2009). Estudo epidemiológico das fraturas do côndilo da mandíbula. Rev. bras. cir. cabeça pescoço, 19-21.

Sever, C., Kulahci, Y., Uygur, F., \& Karagoz, H. (2011). Unusual split fracture of the mandible. Journal of Craniofacial Surgery, 22(6), e10-e11.

Souza, M., Momesso, G., Santos, G., Figueiredo, C., Lima, V., Polo, T., \& Faverani, L. (2018). Tratamento cirúrgico de fratura mandibular atípica em ângulo mandibular com extensão para ramo. Relato de caso. ARCHIVES OF HEALTH INVESTIGATION, 7.

Torres, L. H. S., Uchoa, C. P., Cavalcante, M. B., Jardim, V. B. F., Rodrigues, É. D. R., Pereira, R. V. S., \& Pereira Filho, V. A. (2020). Colles external fixator as alternative in comminuted mandibular fractures treatment. Research, Society and Development, 9(10), e1879108030. doi:10.33448/rsd-v9i10.8030

Ushin, A., Levenets, A., Paniotov, A., \& Pristrom, M. (1988). Mandibular fracture with an atypical complication. Stomatologiia, 67(6), 74-75.

Wubulihasimu, Z., Tuerhong, M., Zhang, Z., Li, H., Kadir, N., Xie, M., \& Ni, Y. (2019). Clinical Analysis and CT 3D-Mediated Precise Internal Fixation in Maxillofacial Fracture. Ear Nose Throat J, 145561319882114. doi:10.1177/0145561319882114 